\title{
Distant galaxy clusters in the COSMOS field found by HIROCS ${ }^{\star}$
}

\author{
M. Zatloukal ${ }^{1}$, H.-J. Röser ${ }^{1}$, C. Wolf ${ }^{2}$, H. Hippelein ${ }^{1}$, and S. Falter ${ }^{3}$ \\ 1 Max-Planck-Institut für Astronomie, Königstuhl 17, 69117 Heidelberg, Germany \\ e-mail: Zatloukal@mpia.de \\ 2 University of Oxford, Department of physics, Denys Wilkinson Bldg., Keble Road, Oxford OX1 3RH, UK \\ 3 I. Physikalisches Institut, Universität zu Köln, Zülpicherstr. 77, 50937 Köln, Germany
}

Received 12 June 2007 / Accepted 24 August 2007

\section{ABSTRACT}

\begin{abstract}
We present the first high-redshift gal axy cluster candidate sample from the HIROCS survey found in the COSMOS field. It results from a combination of public COSMOS with proprietary H-band data on a $0.66 \square^{\circ}$ part of the COSMOS field and comprises 12 candidates in the redshift range $1.23 \leq z \leq 1.55$. We find an increasing fraction of blue cluster members with increasing redshift. Many of the blue and even some of the reddest member galaxies exhibit disturbed morphologies as well as signs of interaction.
\end{abstract}

Key words. surveys - galaxies: clusters: general - galaxies: high-redshift - galaxies: photometry

\section{Introduction}

Galaxy clusters provide ideal laboratories to study galaxy evolution. Furthermore, they can also be used to constrain cosmological parameters (Voit 2005) as well as theoretical models of environmental effects for which there is growing evidence (Khochfar \& Ostriker 2007). Only few galaxy clusters at $z \geq 1$ are known today, most of them stemming from X-ray surveys (Mullis et al. 2005; Stanford et al. 2006). These detect clusters with a hot intra-cluster medium, as will future surveys based on the Sunyaev-Zel'dovich effect (Kneissl et al. 2001). Optical selection of clusters provides a complementary sample (Gilbank et al. 2004), tracing stellar light. At $z \approx 1.1$, where the $4000 \AA$ break gets shifted to the near-infrared, optical surveys need to be supplemented with at least one near-IR band. With the advent of wide-field near-IR cameras such as OMEGA2000 on Calar Alto, wide-area imaging surveys of $z \geq 1$ clusters have become feasible (Elston et al. 2006; Lawrence et al. 2006). A number of optical/near-IR surveys is now beginning to find clusters in this redshift range (Stanford et al. 2005; van Breukelen et al. 2006).

In this Letter we use proprietary $H$-band data in combination with COSMOS public data (Scoville et al. 2006) to find clusters in the redshift range $1.2 \leq z \leq 1.6$ in the COSMOS field. Our detection method employs photometric redshifts to estimate the distances and finds clusters as overdensities in 3D galaxy distribution. Hence, not all our candidates need to be virialized. Some of them may also be clusters in the state of formation. Throughout this letter we assume $H_{0}=70 \mathrm{~km} \mathrm{~s}^{-1} \mathrm{Mpc}^{-1}$, $\Omega_{\mathrm{m}}=0.3$ and $\Omega_{\Lambda}=0.7$. All magnitudes quoted are in the Vega system.

\footnotetext{
* Based on observations collected at the Centro Astronómico Hispano Alemán (CAHA) at Calar Alto, operated jointly by the MaxPlanck-Institut für Astronomie and the Instituto de Astrofísica de Andalucía (CSIC).
}

\section{Data}

HIROCS, the Heidelberg InfraRed/Optical Cluster Survey, aims at finding galaxy clusters at $0.5 \leq z \leq 1.5$ by means of photometric redshifts. The survey area is $11 \square^{\circ}$ in four fields, one of them including the COSMOS field. A detailed description of the survey will be found in Röser et al. (in preparation).

In the COSMOS field, HIROCS uses the public data in $u^{*}$, $B_{j}, g+, V_{j}, r+, i+, N B 816$ and $z+$ (Capak et al. 2007). $K_{\mathrm{s}}$ is used only as a supplement since it is very shallow compared to the other bands. We use the spatially psf homogenized matched_psf mosaics with PSFs from $0.9^{\prime \prime}$ to $1.6^{\prime \prime}$ for the individual filters. The data come calibrated in units of nJy with information about errors and bad pixels stored in separate RMS images. Morphological information is provided by HST ACS $F 814 W$ data (Koekemoer et al. 2007).

As a complement to the optical COSMOS data we use proprietary $H$-band data taken with OMEGA2000 at Calar Alto Observatory in a joint effort of the large extragalactic survey projects ALHAMBRA (Moles et al. 2005), MUNICS-deep (Goranova et al., in preparation) and HIROCS. The COSMOS field will be covered by 25 OMEGA2000 pointings of $15.4^{\prime} \times$ $15.4^{\prime}$ with a targeted depth of $21 \mathrm{mag}(5 \sigma)$ and seeing $<1.6^{\prime \prime}$. Currently, the $H$-band data cover $1.3 \square^{\circ}$ with a non-uniform depth. For this letter we use the deepest $0.66 \square^{\circ}$ which reach 21.4 mag $(3 \sigma)$ or deeper, see Fig. 2.

\section{Data reduction and analysis}

The OMEGA2000 reduction pipeline (Faßbender 2007) was used to reduce the $H$-band data applying object masking for sky determination and image stacking using relative weights and fractional pixel offsets. Flatfields are derived from the science data. Bad pixel and cosmics with a difference of $>3 \sigma$ to a median image are replaced by the scaled median value. The images are astrometrically aligned using point sources from the 2MASS catalogue (Skrutskie et al. 2006). 
Our final object catalog contains all objects from all the bands regardless of their colour with positions as input for the photometry. We use SEXTRACTOR (Bertin \& Arnouts 1996) to extract an object list from the coadded images for each filter with a minimum $\mathrm{S} / \mathrm{N}$ threshold of 5 , omitting the shallow $K_{\mathrm{s}}$ images. Spurious detections, e.g. in the halos around bright stars, are removed manually. Next, an artificial image containing all objects from the different SEXTRACTOR tables is created. For each object, a Gaussian is added into this image with the $\mathrm{S} / \mathrm{N}$ as amplitude and the shape as measured. The final catalog is created by running SE XTRACTOR on this artificial image with a special setup for noise-free data. It reaches a mean positional accuracy of $\sim 0.1^{\prime \prime}$.

We use MPIAPHOT to derive instrumental magnitudes as weighted sums over the image area (Röser \& Meisenheimer 1991). The width of the weighting function is adjusted such that the measurements refer to a common Gaussian "aperture" of 1.8" for all images: $\sigma_{\text {common }}^{2}=\sigma_{\text {weight }}^{2}+\sigma_{\text {seeing }}^{2}$, equivalent to the beam in aperture synthesis radio astronomy. Photometry in the COSMOS bands is done on the coadded frames. The flux errors are measured using the same parameter files on variance frames created by squaring the flux of the RMS frames. $H$-band photometry is done on subsums of 5 one-minute exposures coadded using relative weights and fractional pixel offsets.

The $H$-band needs a mosaic correction of the instrumental magnitudes due to varying observing conditions. Each of the 25 pointings is calibrated using 2MASS stars brighter than $H=14.5$. We get a mean zeropoint error of 0.008 with an RMS of 0.07 measured across the whole field. Fine tuning of the photometric calibration zeropoint, especially to relate the optical COSMOS magnitudes to the infrared magnitudes, is done by applying a stellar main sequence colour shifting technique similar to the one used by Wolf et al. (2001).

Our object catalog comprises 32798 objects with $H<21.4$ to be subject to multi-colour classification. Due to the deep COSMOS optical data, all objects have photometric redshifts assigned and our catalog is essentially complete down to $H=21.4$.

The multi-colour classification and photometric redshift estimation is done using the template-based code developed for CADIS and COMBO-17 by Wolf et al. (2001). The quality of the photometric redshifts is demonstrated in Fig. 1. We use 140 spectroscopic redshifts from Prescott et al. (2006) leaving out the QSOs and a sample of 48 zCOSMOS (Lilly et al. 2006) objects from masks obtained from the ESO archive (Gabasch et al., in preparation) to compare our photometric redshifts with. Both samples are fitted equally well by our code. A Gaussian fit to the $\Delta z /(1+z)$ distribution shows an offset of 0.010 and scatter of $\sigma=0.028$ for $z<1$. We get about $2 \%$ catastrophic outliers with $|\Delta z /(1+z)|>3 \sigma=0.084$. This accuracy is a lower limit at bright magnitudes, while larger deviations are expected for fainter objects. We use a combination of the internally expected error and a lower limit that increases with magnitude and scales with $(1+z)$ as a more conservative error estimate. A $z=24^{\mathrm{mag}}$ galaxy at $z=1.4$ thus has a median error of $\sigma_{z} \sim 0.30$.

A cross-check shows our photo-zs to be in good agreement with the spectroscopic redshifts and the photo-zs from the COSMOS catalog (Mobasher et al. 2006) below $z=0.4$. Our code seems to underestimate redshifts at $0.4 \leq z \leq 1.0$, see upper panel of Fig. 1. At $z \geq 0.75$, the COSMOS catalog redshifts tend to be a little overestimated compared to the spectroscopic sample, leading to a systematic difference of $z \approx 0.08 \ldots 0.1$ with respect to our photo-zs. This trend seems to continue to higher redshifts (see lower panel of Fig. 1).

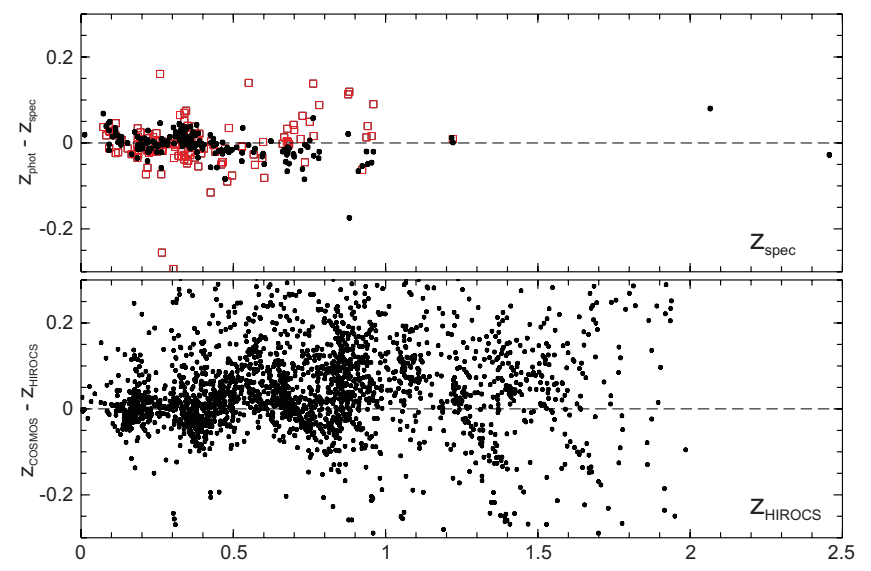

Fig. 1. Upper panel: comparison of spectroscopic and photometric redshifts for HIROCS (filled cirles) and the COSMOS catalog (open squares). Lower panel: comparison of HIROCS and COSMOS photometric redshifts up to $z=2$.

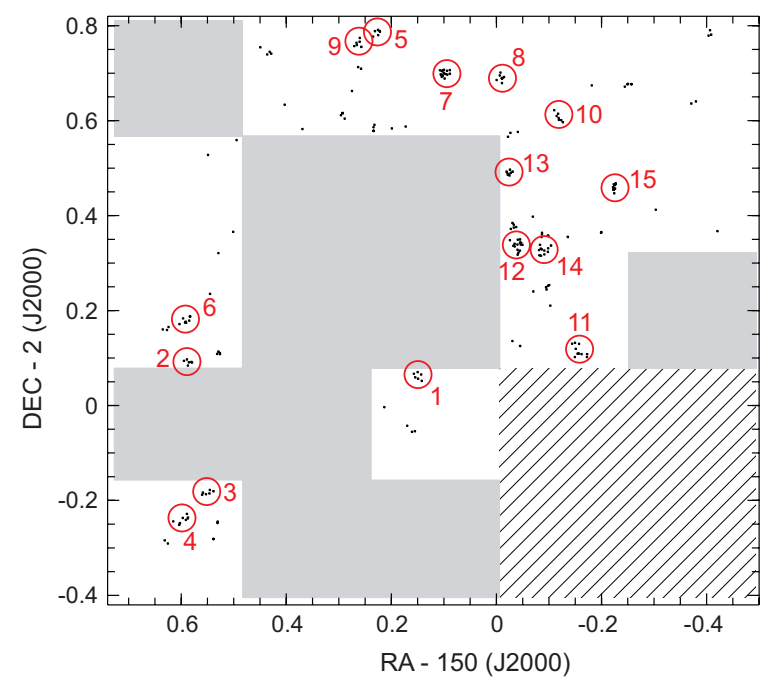

Fig. 2. $H$-band coverage of the COSMOS field and overdense regions in the redshift range $1.2 \leq z \leq 1.6$. In the dashed region there is no $H$-band data at all, in the light grey regions it falls short of our target depth of $H=21.4(3 \sigma)$. The IDs refer to Table 2 .

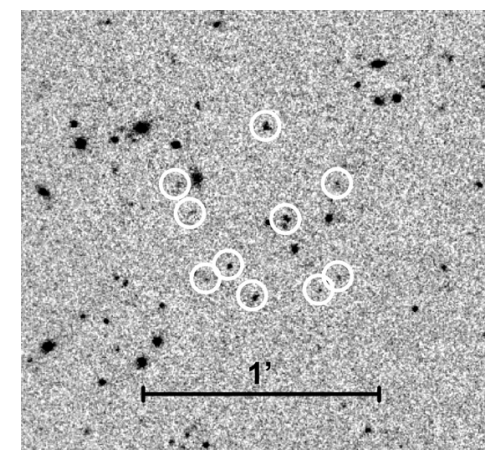

Fig. 3. $H$-band image of candidate HIROCS $095954.2+022924$ (\#13) at $z=1.45$. Member galaxies are marked with white circles.

Rest frame magnitudes are computed by convolving a redshifted filter curve with the best-fitting template spectral energy distribution (SED) normalizing with the nearest observed band.

To find galaxy clusters we use a method based on detecting excess density in the 3D galaxy distribution (Röser et al., in preparation) by computing the local density for all objects. For each object, the fractions of the photometric redshift 


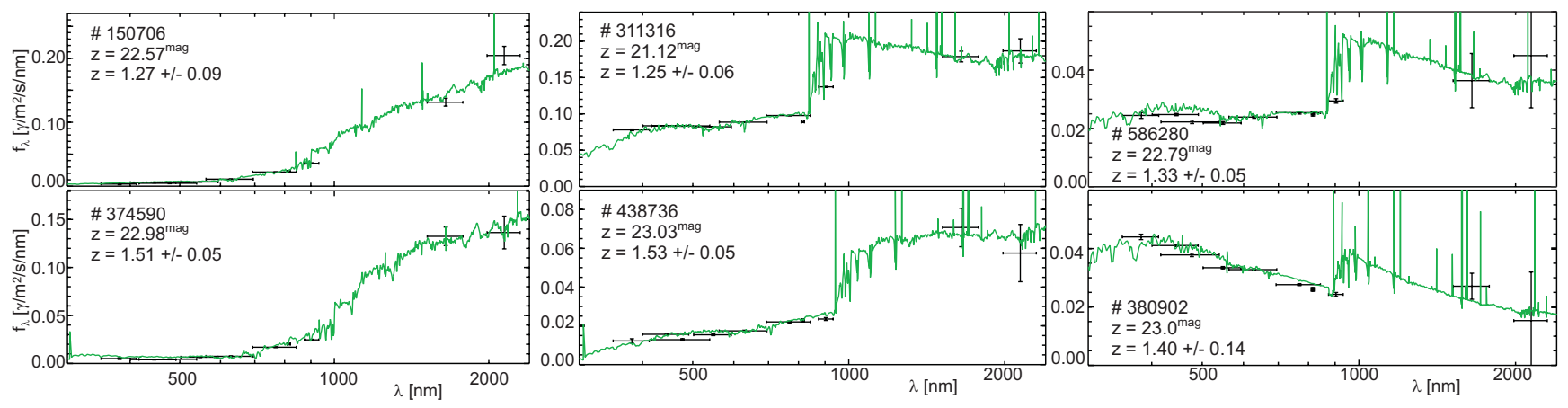

Fig. 4. Representative SEDs of cluster candidate members in two redshift slices. $z \approx 1.25$ in the upper and $z \approx 1.5$ in the lower row. The left column shows very red SEDs whereas the middle and right columns show blue SEDs, indicative of recent or ongoing star formation. The redshift errors quoted are the results of the template fitting algorithm.

Table 1. Correlation of six $z \geq 0.9$ X-ray selected clusters from Table 1 of Finoguenov et al. (2006). ID refers to this table, $z_{\mathrm{XMM}}$ is the redshift given there, $z_{\text {HIROCS }}$ is the photometric redshift found by HIROCS. $M_{500, \mathrm{X}}$ is the mass estimate based on X-ray luminosity, $M_{V, \text { tot }}$ the total rest-frame $V$-band luminosity. $M_{M / L, V}$ is the mass estimate based on $M_{V, \text { tot }}$. Masses are given in units of $10^{13} M_{\odot}$.

\begin{tabular}{cccccc}
\hline \hline ID & $z_{\text {XMM }}$ & $z_{\text {HIROCS }}$ & $M_{500, \mathrm{X}}$ & $M_{V, \text { tot }}$ & $M_{M / L, V}$ \\
\hline 32 & 0.90 & 0.79 & $18.64 \pm 0.52$ & -25.0 & 12.0 \\
66 & 0.95 & - & $1.81 \pm 0.45$ & - & - \\
72 & 0.90 & 0.84 & $5.34 \pm 0.47$ & -23.6 & 3.2 \\
102 & 1.10 & 0.93 & $2.60 \pm 0.38$ & -24.5 & 7.4 \\
126 & 1.00 & 0.86 & $6.87 \pm 0.69$ & -24.3 & 6.1 \\
133 & 1.15 & - & $5.68 \pm 0.64$ & - & - \\
\hline
\end{tabular}

probability distributions $p(z)$ of all its neighbours within $300 \mathrm{kpc}$ in projected physical separation and a $\Delta z$ motivated by the average accuracy of our photometric redshifts are added. The resulting densities turn out to be rather insensitive to the exact value of $\Delta z$. Only objects with $3 \sigma$ overdensity compared to the average field are used to select cluster candidates. All structures with more than 6 overdense members within an aperture of $2^{\prime}$ are picked as cluster candidates from a two-dimensional plot of overdense galaxies in redshift slices to avoid projection effects. Figure 2 shows such a plot for the redshift range $1.2 \leq z \leq 1.6$ with the cluster candidates highlighted.

Monte-Carlo simulations with COMBO-17 data have shown this $3 \sigma$ overdensity limit to be rather conservative (Falter et al., in preparation). Comparison with the density maps of Scoville et al. (2006) at $z<1.1$ shows our overdense objects to trace all density peaks. A correlation of our HIROCS results with Xray selected clusters at $z \geq 0.9$ from Finoguenov et al. (2006) in our selection area shows that four out of six clusters are in common (see Table 1). The difference in redshift results from the different photo-z codes, see Fig. 1. For X-ray cluster 66 we find no counterpart in our $H$-selected object list. While this is a low-mass structure, $\mathrm{X}$-ray cluster 133 is also not detected by our algorithm since it has no members with a local overdensity $>3 \sigma$. Only few galaxies at its position are visible even if we lower the overdensity cut to $1 \sigma$, despite its mass being above the mass of cluster 102 which we detect. All these X-ray selected clusters have redshifts $z_{\text {HIROCS }}<1.2$ and are thus not part of our sample.

\section{Results and discussion}

The first HIROCS cluster candidate sample for the COSMOS field comprises 15 galaxy cluster candidates with redshifts $1.22 \leq z \leq 1.55$. They are listed in Table 2 with their positions and properties. Their significance with respect to the mean
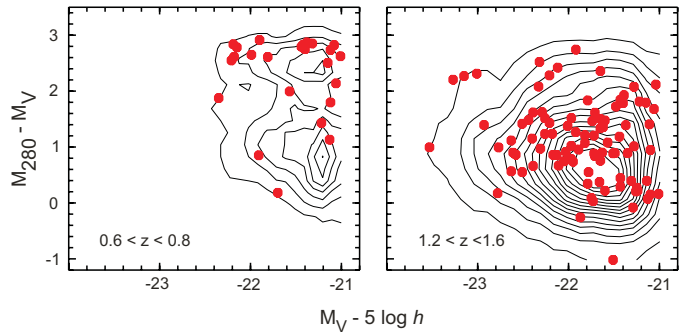

Fig. 5. The restframe $M_{280}-M_{V}$ colour-magnitude diagram in the redshift slices [0.6, 0.8] (left) and [1.2, 1.6] (right). Field galaxies are indicated as contours. Overplotted in red are the members of a known largescale structure at $z \approx 0.7$ (left) and the HIROCS cluster candidates from Table 2 (right).

number density of galaxies in this redshift slice is between $2.4 \sigma$ and $11.4 \sigma$. Candidates \#1 and \#11 are removed from the final list due to their low significance, candidate \#7 because it does not show a clear redshift peak and colour-magnitude plots show it to be most likely a chance projection of two structures. This leaves 12 candidates in the final list. The number of cluster members is limited by the depth of the $H$-band data. Figure 3 shows an $H$-band image of HIROCS 095954.2+022924 (\#13) at $z=1.45$ as an example. Most member galaxies are clustered in a region of about $1 \square^{\prime}$ on the sky.

Some representative cluster members' SEDs from the template fitting process are shown in Fig. 4. Note how the $z$-band flux drops when moving up in redshift from $z=1.2$ to $z=1.4$ (center column), placing a secure lower limit on the photometric redshift. While we observe the very red, passively evolving galaxies expected to be present in galaxy clusters, there is a remarkably high fraction of galaxies with blue SEDs. This is also evident in colour-magnitude diagrams of the cluster candidates. Figure 5 shows restframe $M_{280}-M_{V}$ colour-magnitude diagrams of all galaxies with $H \leq 21.4$ in the redshift slices $[0.6,0.8]$ (left) and $[1.2,1.6]$ (right). Overplotted in red are the members of a rich large-scale structure at a redshift of $z \approx 0.7$ (Guzzo et al. 2007) (left panel) and members of the cluster candidates given in Table 2 (right panel). The strength of the colour bimodality decreases with redshift, as observed for example by Cirasuolo et al. (2007). The fraction of red galaxies is considerably lower in our high-redshift candidates than in the $z=0.7$ structure. This could be due to a Butcher-Oemler type evolution, a colour-density effect or a combination of both.

On the HST ACS images many members exhibit disturbed morphologies and signs of interactions. This is seen for a lot of the blue and even for some of the reddest galaxies. Note that the term "red" only refers to colour and includes both passively evolving old stellar populations as well as dust-reddened objects. 
Table 2. First sample of HIROCS clusters in COSMOS with $z \geq 1.2$. An ID marked with “*” means that this candidate was rejected from the final list, see text for details. The cluster redshift $z$ is the mean redshift of its members, $\sigma_{z}$ the error of the mean. $N_{>3 \sigma}$ contains the number of members above the $3 \sigma$ overdensity cutoff without correction for field galaxies, whereas $N_{\text {tot }}$ gives the total number of galaxies at the cluster redshift and position regardless of their local density with the average field density subtracted. They are selected to be within a circular aperture of $2 \times$ the median distance of the overdense members to the cluster center and to be in the redshift slice $z_{\text {cluster }} \pm 0.15$. The significance of $N_{\text {tot }}$ with respect to the mean field density is given in "significance". $M_{V, \text { BCG }}$ gives the total restframe $V$-band luminosity of the brightest cluster galaxy, $M_{V, \text { tot }}$ the field corrected total luminosity in the restframe $V$-band down to the completeness limit. No correction for completeness is applied to $M_{V \text {,tot }} M_{M / L, V}$ gives a mass estimate based on $M_{V, \text { tot }}$, see text for details. Our $50 \%$ completeness limits are $M_{V}=-21.4$ at $z=1.3$ and $M_{V}=-21.8$ at $z=1.5$.

\begin{tabular}{cccccccccccc}
\hline \hline ID & Name & $\begin{array}{c}\left.\text { RA [ }{ }^{\circ}\right] \\
(\mathrm{J} 2000)\end{array}$ & $\begin{array}{c}\text { Dec }\left[{ }^{\circ}\right] \\
(\mathrm{J} 2000)\end{array}$ & $z$ & $\sigma_{z}$ & $N_{>3 \sigma}$ & $N_{\text {tot }}$ & $M_{V, \text { BCG }}$ & $M_{V, \text { tot }}$ & $\begin{array}{c}\text { Significance } \\
{[\sigma]}\end{array}$ & $\begin{array}{c}M_{M / L, V} \\
{\left[10^{13} M_{\odot}\right]}\end{array}$ \\
\hline $1^{*}$ & HIROCS 100035.6+020338 & 150.148 & 2.061 & 1.22 & 0.03 & 7 & 6 & -23.2 & -23.9 & 2.4 & 2.9 \\
2 & HIROCS 100220.5+020534 & 150.586 & 2.093 & 1.23 & 0.07 & 9 & 8 & -22.6 & -24.2 & 4.5 & 4.0 \\
3 & HIROCS 100212.2+014858 & 150.551 & 1.816 & 1.24 & 0.06 & 9 & 11 & -23.8 & -25.0 & 5.3 & 7.9 \\
4 & HIROCS 100223.7+014527 & 150.599 & 1.758 & 1.24 & 0.06 & 10 & 11 & -23.7 & -24.5 & 3.5 & 5.0 \\
5 & HIROCS 100054.5+024703 & 150.227 & 2.784 & 1.26 & 0.06 & 7 & 12 & -24.0 & -24.8 & 9.0 & 6.5 \\
6 & HIROCS 100221.8+021045 & 150.591 & 2.179 & 1.31 & 0.05 & 8 & 10 & -23.3 & -24.8 & 6.4 & 6.1 \\
$7 *$ & HIROCS 100023.9+024158 & 150.100 & 2.699 & 1.32 & 0.09 & 17 & 16 & -23.1 & -25.2 & 9.1 & 8.8 \\
8 & HIROCS 095957.9+024125 & 149.991 & 2.690 & 1.33 & 0.06 & 8 & 10 & -23.4 & -24.9 & 7.3 & 6.2 \\
9 & HIROCS 100103.2+024546 & 150.263 & 2.763 & 1.40 & 0.12 & 6 & 8 & -23.5 & -24.7 & 6.6 & 4.7 \\
10 & HIROCS 095931.8+023630 & 149.882 & 2.608 & 1.41 & 0.04 & 6 & 8 & -23.1 & -24.7 & 4.4 & 4.7 \\
$11 *$ & HIROCS 095922.3+020654 & 149.843 & 2.115 & 1.43 & 0.04 & 10 & 19 & -23.4 & -25.8 & 2.5 & 13.1 \\
12 & HIROCS 095950.3+022014 & 149.960 & 2.337 & 1.44 & 0.07 & 15 & 19 & -24.3 & -25.9 & 8.4 & 14.3 \\
13 & HIROCS 095954.2+022924 & 149.976 & 2.490 & 1.45 & 0.05 & 8 & 10 & -23.6 & -24.9 & 8.0 & 5.2 \\
14 & HIROCS 095938.4+021939 & 149.910 & 2.328 & 1.52 & 0.06 & 14 & 18 & -23.0 & -25.3 & 7.7 & 6.8 \\
15 & HIROCS 095906.0+022726 & 149.775 & 2.457 & 1.55 & 0.05 & 8 & 8 & -23.2 & -23.9 & 11.4 & 1.7 \\
\hline
\end{tabular}

The total rest-frame $V$-band luminosity $M_{V \text {,tot }}$ of the candidates is in the range $-25.8 \leq M_{V \text {,tot }} \leq-24.2$ with the brightest cluster galaxies having rest frame luminosities in the range $-24.3 \leq M_{V, \mathrm{BCG}} \leq-22.6$. The brightest of these are consistent with present-day BCGs (Bahcall 2000) when applying a passive evolution correction factor based on the evolution of $M_{B}^{*}$ from Bell et al. (2004) with $z_{\text {form }}=3$. Our mass estimates $M_{M / L, V}$ based on the total rest-frame luminosity are in good agreement with the X-ray based $M_{500}$ masses, see Table 1 . We assume massto-light ratios of $140(z=0.9)$ and $75(z=1.4)$ based on the present-day mass-to-light ratio of 300 (Bahcall 2000) and the correction for evolution described above. Comparison with the mass estimates of the X-ray selected catalog shows that our algorithm detects structures down to at least $M_{500, X}=2.6 \times 10^{13} M_{\odot}$, the mass of X-ray selected cluster 102 , at $z=0.95$. Our lowestmass $z \geq 1.2$ candidate HIROCS $095906.0+022726$ (\#15) has a total mass of $M_{M / L, V} \approx 1.7 \times 10^{13} M_{\odot}$. At present we do not have a completeness limit estimate for our algorithm. Theoretical models $\left(\Lambda \mathrm{CDM}, \sigma_{8}=0.8\right)$ predict about 12 clusters per $0.66 \square^{\circ}$ at $z>1.1$ with $M \geq 5 \times 10^{13} M_{\odot}$ (Bartelmann \& White 2002; M. Bartelmann priv. comm.). If we take $M_{200}=1.7 \times M_{500}$ (Finoguenov et al. 2006) and assume $M_{500}=M_{M / L, V} 11$ of our 12 candidates fall into this mass range, matching the predictions. Note that no contamination or completeness corrections have been applied. The cluster candidates we find are comparable in redshift to the most distant spectroscopically confirmed (McCarthy et al. 2007) and photometrically selected (Castellano et al. 2006) clusters known today. These results indicate that with the full HIROCS galaxy cluster sample implications on cosmological parameters and galaxy evolution will become assessible.

Acknowledgements. The authors would like to thank the COSMOS team for making their data publicly accessible, R. Faßbender for providing the IR reduction pipeline and the staff at Calar Alto Observatory for their support. The data were taken in a joint effort with the MUNICS-deep team (U. Hopp, MPE Garching) and the ALHAMBRA team (M. Moles, IAA Granada). It is a pleasure to thank both teams for the kind collaboration. The authors would also like to thank S. Khochfar for valuable comments on the draft version of this letter, I. Sakelliou for helpful discussions on the X-ray data and S. Noll (MPE Garching) for sharing his spectroscopic comparison sample. This publication makes use of the NASA ADS and data products from the Two Micron All Sky Survey, as well as of the NASA/ IPAC Infrared Science Archive. We thank an anonymous referee for a thorough and very helpful report.

\section{References}

Bahcall, N. 2000, Allen's Astrophysical Quantities, 4th Ed., ed. A. N. Cox (New York: Springer Verlag)

Bartelmann, M., \& White, S. D. M. 2002, A\&A, 388, 732

Bell, E. F., Wolf, C., Meisenheimer, K., et al. 2004, ApJ, 608, 752

Bertin, E., \& Arnouts, S. 1996, A\&AS, 117, 393

Capak, P., Aussel, H., Ajiki, M., et al. 2007, [arXiv : 0704 .2430]

Castellano, M., Salimbeni, S., Trevese, D., et al. 2006, ArXiv e-prints

Cirasuolo, M., McLure, R. J., Dunlop, J. S., et al. 2007, ArXiv e-prints

Elston, R. J., Gonzalez, A. H., McKenzie, E., et al. 2006, ApJ, 639, 816

Faßbender, R. 2007, Ph.D. Thesis, Ludwig-Maximilians-Universität München

Finoguenov, A., Guzzo, L., Hasinger, G., et al. 2006,

[arXiv: astro-ph/0612360]

Gilbank, D. G., Bower, R. G., Castander, F. J., \& Ziegler, B. L. 2004, MNRAS, 348,551

Guzzo, L., Cassata, P., Finoguenov, A., et al. 2007,

[arXiv: astro-ph/0701482]

Khochfar, S., \& Ostriker, J. P. 2007, [arXiv: 0704.2418]

Kneissl, R., Jones, M. E., Saunders, R., et al. 2001, MNRAS, 328, 783

Koekemoer, A. M., Aussel, H., Calzetti, D., et al. 2007, [arXiv: astro-ph/0703095]

Lawrence, A., Warren, S. J., Almaini, O., et al. 2006,

[arXiv: astro-ph/0604426]

Lilly, S. J., Le Fevre, O., Renzini, A., et al. 2006, [arXiv: astro-ph/0612291]

McCarthy, P. J., Yan, H., Abraham, R. G., et al. 2007,

[arXiv: astro-ph/0701787]

Mobasher, B., Capak, P., Scoville, N. Z., et al. 2006, ArXiv Astrophysics e-prints

Moles, M., Alfaro, E., Benítez, N., et al. 2005, [arXiv: astro-ph/0504545]

Mullis, C. R., Rosati, P., Lamer, G., et al. 2005, ApJ, 623, L85

Prescott, M. K. M., Impey, C. D., Cool, R. J., \& Scoville, N. Z. 2006, ApJ, 644, 100

Röser, H.-J., \& Meisenheimer, K. 1991, A\&A, 252, 458

Scoville, N., Aussel, H., Benson, A., et al. 2006, [arXiv: astro-ph/0612384]

Skrutskie, M. F., Cutri, R. M., Stiening, R., et al. 2006, AJ, 131, 1163

Stanford, S. A., Eisenhardt, P. R., Brodwin, M., et al. 2005, ApJ, 634, L129

Stanford, S. A., Romer, A. K., Sabirli, K., et al. 2006, ApJ, 646, L13

van Breukelen, C., Clewley, L., Bonfield, D. G., et al. 2006, MNRAS, 373, L26

Voit, G. M. 2005, Rev. Mod. Phys., 77, 207

Wolf, C., Meisenheimer, K., Röser, H.-J., et al. 2001, A\&A, 365, 681 